est advances, but if we sift down the facts will we not find that those advances have been more in the line of possible mutilations than in the cure of disease? The successful removal of a woman's stomach in its entirety is undoubtedly a great surgical feat, but was not its removal caused by a confession on the part of the surgeon of his inability to cure the disease. Far be it from me to in any way minimize the untold benefits which have been enjoyed by suffering humanity, as a result of the hard work, the deep study and the combined efforts of all who belong to the medical profession, and to be a member of which I consider myself honored, but it seems to me that it is sometimes healthful to stop and consider what we are really doing; not to rest too comfortably on our laurels already obtained, but by a just and honest view of our possible shortcomings, to remember that there are new fields to be cultivated. new glory to be won in our everlasting battle with disease, and that each one of us, however humble we may be, may by diligent work, thought and observation, be able to add our mite to that grand sum which makes up the total of human knowledge.

\section{A RATIONAL METHOD OF RELIEVING ASPHYXIA IN THE NEW-BORN INFANT.}

Read before the Florida Medical Association, April 27, 1898.

BY S. STRINGER, M.D.

$$
\text { BROOKSVILLE, FLA. }
$$

I desire to call the attention of the medical profession to a method of relieving asphyxiated new-born infants which I do not think has been presented to their notice heretofore.

I do not claim for this method that it is novel, unique, etc., but that it is based entirely upon chemic and philosophic principles and, like many other facts in our profession, is the result of accidental discovery.

We know that in the asphyxiated infant, unless the blood becomes oxygenated very soon, the child must die; we know that from several causes the sensorium has become so deadened or blunted as not to respond to the irritation of the atmospheric air, the application of water or other methods of inducing respira. tion; yet fetal life still remains and would continue were it not that the placenta has become detached and thereby respiration or oxygenation of the blood, through the medium of maternal circulation, is cut off. Now, if we could maintain fetal life by any method until the sensorium can sufficiently recover to respond to the nervous excitants of respiration we would, in many instances, save the life of the infant.

How this is to be done is the object of this short essay, as well as to relate the circumstances which led to the discovery thereof.

A few years ago I was called to see a multipara in labor and was told she was in about her fifth month of pregnancy. In a few hours she was delivered of a fetus which I thought to be close to the age indicated. The fetus, membranes and placenta were all delivered by the same effort. Nothing unusual having occurred, the fetus and envelope were laid aside till my departure, when $I$ had it placed in some cloths and rolled up to carry with me for the purpose of saving it as a specimen, but, it being late at night, I deposited it till morning, when I proceeded to examine it and, to my astonishment, found the fetal circulation still going on, with pulse at wrist very perceptible. This was several hours after birth.
Here was a case of fetal circulation, to my mind, carried on by the aeration of the blood through the medium of the placenta exposed to the atmospheric air.

In contemplating this case it occurred to me that this information might be utilized in cases of asphyxia in new-born infants, and I resolved to try the first case that came under my care.

At last a case presented itself; a large and welldeveloped child, of white parentage, and in which the head had been molded into cylindrical form by a narrow pelvis. The child could not be induced, by the usual method of cold application and rolling, to make any effort at respiration. The circulation was still going on in the funis with some vigor, but the deep. ening of the dark hue of the surface plainly indicated that, unless oxygenation of the blood could take place, death would soon follow.

Already the pulsation in the cord had become feeble and was becoming more so rapidly, when I delivered the placenta, cleaned it of clots and exposed the maternal surface to the atmospheric air. In a very short time the pulsation was perceptibly increasing in force; the livid and death.like hue was being displaced by one of life and health, when it required but a fow moments for the restoration of sensibility and process of respiration commenced.

I feel sure that had the placenta remained in the os uteri or vagina, excluded as it was from atmospheric air, death from asphyxiation of the child would certainly have occurred.

Several subsequent cases have proven, to my mind, that this simple and rational plan of restoration is preferable to all others; and I will ask, Why not? Does not the blood become rapidly oxygenated when exposed to the air even in an open vessel? Have you not seen venous blood reddened in a fow moments after exposure to atmospheric air? Then, why not when exposed to the air by osmosis, through the irregular surface of the placenta, where aeration had been going on from the time of the earliest distinct organization of the embryo?

In nearly all cases of asphyxia the labor has been tedious, owing to narrow passage and time to mold the head to the same by the powerful expulsive effort which detaches the placenta and throws it into the os, or entirely into the vagina, as the child emerges from the third stage of labor. Therefore you will find no difficulty in the immediate removal of placenta, should you have a case of asphyxia.

After its removal from the vagina it will be much easier to lift the cord from the neck and examine it for any interference with free circulation and correct the same. The placenta should be spread out with maternal surface cleansed of all clots and membranes so that free access of air can be had. If it becomes necessary, on account of numerous clots, to use water to cleanse the maternal surface, it is advisable to have it warm, as it is remarkable how quickly the use of cold water will chill the child; yet it is but natural to expect such result when you apply cold to so large surface of the capillary circulation as is maintained in the maternal surface of the placenta.

So long as the circulation keeps on through the cord you need not fear for the life of the child, for it is a continuance of fetal life, after birth

As soon as respiration occurs, which has been delayed as long as twenty-five minutes in some of my cases, the circulation is diverted from the placenta to the lungs, and pulsation in the cord ceases in a few 
seconds, when the child should be separated from the placenta as in ordinary cases.

I commend this method to the profession and ask that it be tried where more cases present themselves than are met with by provincial practitioners, when, I am convinced, it will be universally adopted.

I can not omit referring to the great advantages which, it seems to me, must result from the delivery of the placenta in cases of placenta previa, advantages not only to the mother but to the offspring. As the consideration of this subject would require considererable time I will only give a fow extracts from the literature on dystocia. Dr. Simpson has collected 120 cases in which the placenta was delivered prior to the birth of the child. Of this number 31 of the children were born alive, 2 were putrid and 87 still-born. I have no doubt but that a large per cent. of the 87 still-born died of asphyxia, in consequence of the retention of the placenta for some time after its detachment. Had it been promptly delivered and exposed to atmospheric air many lives would have been saved.

\section{ALBUMINURIA FROM THE STANDPOINT OF LIFE INSURANCE}

BY MARK A. BROWN, M.D.

CLINICAL LACTURER ON MEDICINE, MIAMI MEDICAL COLLEGE. CINCINNATI, OHIO.

At the present day, in considering the advisability of accepting a certain risk, the presence or absence of albumin in the urine may be readily regarded as the deciding weight, either for rejection if present or acceptance if not. Certain it is that the examination of the urine for this abnormal ingredient is looked upon by the home office as of such importance that except in industrial insurance, no report will be accepted without definite statements. Nor is this a matter of surprise when we remember the large number of fatal diseases accompanied by albuminuria. In many companies the albuminuria alone is sufficient cause for rejection and the underlying disease, if not entirely disregarded, is at least looked upon as a mat. ter of but small moment. In this manner mistakes are made upon both sides. No greater error can be made than to look upon every case of albuminuria as Bright's disease, unless it is to regard every case not accompanied by albuminuria as not being Bright's.

It has been estimated that about one-sixth of the applications for life insurance (ordinary) are refused, all causes being taken into consideration. At least one-fourth of these rejections are based upon the presence of albumin in the urine. It is preposterous to assume that one twenty-fourth of the number that apply for life insurance are afflicted with nephritis; we must always be on the lookout for other explanations than irreparably damaged kidneys to account for our symptoms. Nor must it be forgotten that albumen may exist in the urine in several forms, of which serum albumin is the most important, responding to the usual rough tests applied by examiners. Again an admixture of urine with substances containing albumin, blood, seminal and purulent fluid, will throw down a white cloud to the heat and nitric acid test just as readily as will a chemical solution of albumin in urine. Here enters a first plea for microscopic examination in all cases where the slightest trace of albumin is found. A small amount of any of the above abnormal adnixtures might and, as a matter of clinical observation, frequently does escape detection on macroscopic examination, yet even such a small amount may cause a slight albuminous reaction, a reaction very like that of chronic interstitial nephritis, the disease which so slowly but surely carries off such immense numbers of the race. One's doults as to the existence of such abnormal constituents as pus cells, blood corpuscles or spermatozoa in the specimen under consideration, will be at once dis. pelled by a few minutes use of the centrifuge and microscope. When we reflect upon the large number of pathologic conditions which might result in the pouring out of blood or pus, or both, into some part of the genito-urinary tract, especially when we remember the great prevalence of urethritis, simple and specific, acute and chronic, we are not much surprised at the frequency of albuminuria; indeed, we are rather inclined to feel correspondingly encouraged at the lessening of the number of cases of possible Bright's. Of late years the increasing frequency of life insurance among women, while not liable to increase the average of insurance mortality of Bright's, yet certainly must hold its own us regards the ratio of albuminuria. Menstruation. endometritis and vaginitis, would easily maintain the standard. From this it can readily be deducted that uranalysis in a female is not of much value unless the urine is obtained by means of the catheter.

Albuminuria in its present day aspect is divided by clinicians into two types, the physiologic and the pathologic. The former is a convenient head under which to class cases in which the symptom is transient or intermittent, and in which it occurs, presenting no other sign of kidney or circulatory disease. The albuminuria of pregnancy is termed by the adherents of this class as physiologic in a large number of cases; that it is a frequent symptom among women in this condition is admitted by all who have looked closely into the question, Aufrecht reporting as high as 56 per cent In new. horn children albuminuria is common during the first few days of life, but the changes in circulation would readily account for this. Among these intermittent cases, the albuminuria that occurs in bicycle riders is of late receiving deserved attention. Mueller ${ }^{1}$ reports eight cases in trained cyclists, in whom, after a fatiguing ride, there appeared an albuminuria which even with the aid of the microscope was absolutely indistinguishable from that of true nephritis. It is interesting to note that after several days of rest the albuminuria disappeured. The urine when examined previous to the ride was free from albumin in all cases but one while after the ride seven out of the eight showed albuminuria and the majority casts of various kinds. These cases will be referred to again in speaking of the pathologic type. Suffice it to say here that they were regarded as physiologic, by their reporter, on the grounds that the albuminuria was intermittent, disappearing completely on the cessation of the excessive exertion, without apparent damage having been sustained by the kidneys. The ultimate fate of these men might make an interesting contribution to the etiology of nephritis. Vaccination is not infrequently followed by transient albuminuria in subjects who were previously free from the abnormality. And so we could go on adding case after case called physiologic because the albuminuria is transient and is not accompanied by easts. Tyson, ${ }^{2}$ in considering physio-

1 Muench. med. Woch., 1896

2 Tyson's Practice of Medicine. 